\title{
EXPANSÃO PARADOXAL DE LESÕES DE TUBERCULOSE CEREBRAL DURANTE $O$ USO DE TUBERCULOSTÁTICOS
}

\author{
ANTÔNIO DE SOUZA ANDRADE FILHO*, ANDRÉ GUANAES GOMES**, \\ ANTONNIO CARLOS MOREIRA DE LEMOS ${ }^{* * *}$ MARGARIDA COSTA NEVES****, \\ YURI MASCARENHAS DE ANDRADE SOUZA*****, SAMIRA LUISA DOS APÓSTOLOS PEREIRA*****, \\ ANDREIA PELTIER DE QUEIROZ URBANO DE SOUZA***** ,PATRICIA LAGO DOS SANTOS*****
}

\begin{abstract}
RESUMO - O acometimento do sistema nervoso central pela tuberculose pode se manifestar de duas formas principais: tuberculose meníngea ou tuberculoma. Esta forma é uma condição que vinha decrescendo progressivamente, mas que encontrou nos dias atuais condições ideais para o seu desenvolvimento. O presente estudo discorre sobre três pacientes que desenvolveram expansão paradoxal de lesões de tuberculose cerebral durante o uso de tuberculostáticos, o que demonstra ser importante o acompanhamento tomográfico de pacientes que apresentem sinais neurológicos no curso do tratamento para a tuberculose. Os dois primeiros pacientes apresentaram o acometimento neurológico associado a tuberculose pulmonar, enquanto no terceiro havia história de tuberculose meníngea prévia. A tomografia computadorizada foi o exame utilizado para o acompanhamento desses pacientes. O tratamento constou do uso de tuberculostáticos e corticóide.
\end{abstract}

PALAVRAS-CHAVE: tuberculoma, tuberculostáticos, tomografia computadorizada.

\section{Paradoxical expansive lesions of cerebral tuberculosis during tuberculostatic drug therapy}

ABSTRACT - Central nervous system involvement by tuberculosis presents two main types: tuberculosus meningitis or tuberculoma. This second condition has nowadays ideal conditions for development. We report three patients who developed paradoxical brain tuberculosis expansive lesion during the use of tuberculostatic drugs. The cases point out the importance of tomography follow up of patients who present neurological signs in the course of treatment for tuberculosis. The first two patients presented onset of the a neurological symptoms, associated with lung tuberculosis. The third patient previously had tuberculous meninigitis. Computerized tomography was used to follow up these patients. Tuberculostatic and corticoid drugs were used for treatment.

KEY WORDS: tuberculoma, tuberculostatic drugs, computerized tomography.

Neurotuberculose, que é a tuberculose do sistema nervoso central (SNC), particularmente meningite tuberculosa ou tuberculoma, resulta de mecanismo complexo em que operam mudanças multifatoriais em toda estrutura e composição celular do cérebro, cerebelo e cordão espinhal, que podem ser detectadas por microscopia eletrônica ou ao exame de peças à macroscopia. Na virada do século, as lesões cerebrais tuberculosas constituiam um terço de todos os tumores cerebrais na

Hospital Otávio Mangabeira / Secretaria da Saúde do Estado da Bahia, Departamento de Neuropsiquiatria (DNP) da Faculdade de Medicina (FAMED) da Universidade Federal da Bahia (UFBA); Fundação de Neurologia e Neurocirurgia, Instituto do Cérebro (FNN/IC): *Professor Titular de Neurologia da Escola de Medicina e Saúde Pública (EMSP) da Fundação para o Desenvolvimento das Ciências. Professor Adjunto Doutor do DNP da FAMED/UFBA; **Chefe da Unidade de Terapia Intensiva do Hospital Santo Amaro; ***Professor Adjunto do Departamento de Medicina Interna do Hospital Universitário Professor Edgar Santos (HUPES); *****Professora Auxiliar de Ensino do Departamento de Medicina do Interna HUPES, Pneumologista; *****Acadêmico de Medicina FAMED/UFBA, Estagiário da FNN/IC. Aceite: 17-fevereiro-1999.

Dr. Antônio de Souza Andrade Filho - Rua Deocleciano Barreto 10 - 40150-400 Salvador BA - Brasil. FAX 071332 2333. E-mail: fundação.neurologia.neurocirurgia@svn.com.br 
Inglaterra ${ }^{1}$. Com a melhora das condições sócio-econômicas e com o advento da quimioterapia, a incidência da tuberculose vinha decrescendo progressivamente, principalmente nos países desenvolvidos, mas atualmente, na era da AIDS, não apenas a evolução de multirresistência a drogas, mas também o estado de imunodeficiência desses pacientes têm sido campo propício para a larga disseminção da tuberculose no mundo ${ }^{2}$. Em 1940, os tuberculomas representavam 3,6\% de 2190 tumores cerebrais, caindo para $0,15 \%$ de $2200 \mathrm{em} 1972^{1}$. Atualmente oscilam entre 0,5 a $1 \%$ nos países de primeiro mundo, enquanto nos países subdesenvolvidos as taxas continuam elevadas, representando 15 a $20 \%$ de todos os tumores cerebrais. É mais comumente encontrado em crianças e adultos jovens após infecção pulmonar primária, a exemplo do que ocorre na Índia, onde $86 \%$ dos pacientes têm idade abaixo dos 20 anos. Em países desenvolvidos como os EUA, ocorre mais em idosos, como resultado da reativação de foco quiescente. Bhargava e col. mostraram que $10 \%$ dos processos de tuberculose meningea evoluem para lesões cerebrais ${ }^{3}$.

Das lesões tuberculosas da SNC, os tuberculomas são mais frequentes que os abscessos e diferenciam-se destes por serem mais ricos na reação granulomatosa, enquanto os últimos apresentamse como cavidade com a parede pobre em reação granulomatosa e preenchida por material necrótico ${ }^{1,3}$. Em 25 a 50\% dos casos de tuberculose cerebral, existe alteração prévia do RX de tórax, compatível com doença em atividade, podendo chegar a $83 \%$ no caso de países subdesenvolvidos, a exemplo da Índia, Chile e Brasil ${ }^{1,4}$. A tomografia computadorizada (TC) de crânio, é o melhor método para acompanhamento desses pacientes, com sensibilidade em torno de $83 \%$, porém, por si própria, não permite a diferenciação de tuberculoma e abcesso cerebral ${ }^{1,3}$. A terapêutica medicamentosa com tuberculostáticos e corticóide (nos casos em que existe hipertensão intracraniana) resolve a maioria dos casos, ficando reservada à cirurgia os casos de falência terapêutica ${ }^{3,4}$.

Existem poucos casos relatados na literatura em que, após iniciado o uso de tuberculostáticos, parodoxalmente à melhora clínica, houve aumento das lesões cerebrais com manutenção ou piora dos sintomas neurológicos, que regrediram após manutenção dos tuberculostáticos associados a corticoterapia ${ }^{2}$. Nós descrevemos três casos em que observamos este fenômeno de expansão paradoxal, dos quais dois apresentavam tuberculose pulmonar associada e o outro, história de tuberculose meníngea prévia.

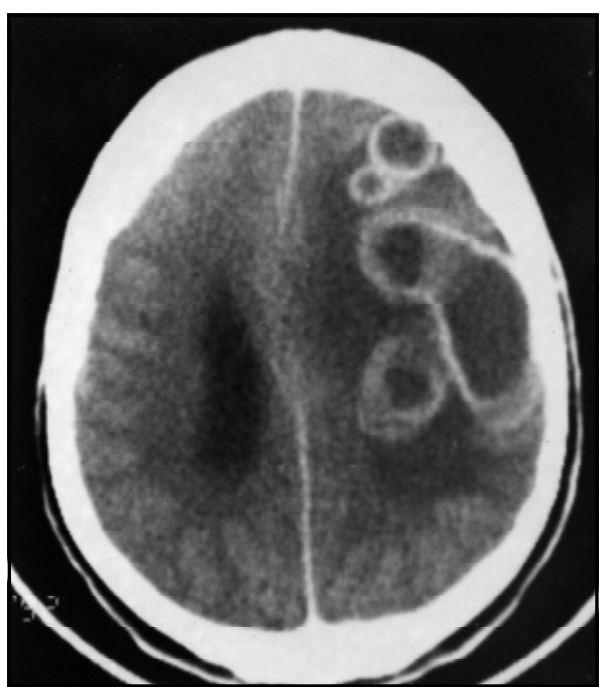

Fig 1. Caso 1. TC de crânio (24/01/86) mostrando lesões hipodensas com halo captante e edema perilesional em região parieto-frontal de hemisfério esquerdo

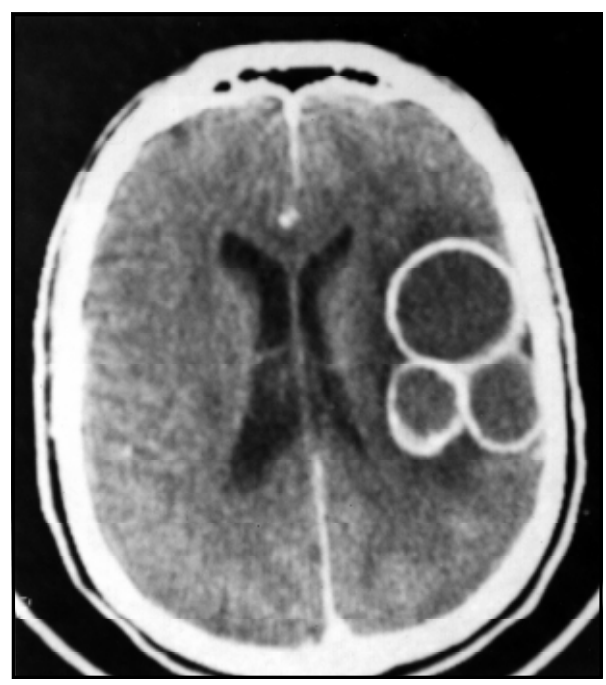

Fig 2. Caso 1. TC de crânio (01/10/86) mostrando desaparecimento das lesões frontais e aumento das lesões parietais em vigência de tratamento. 


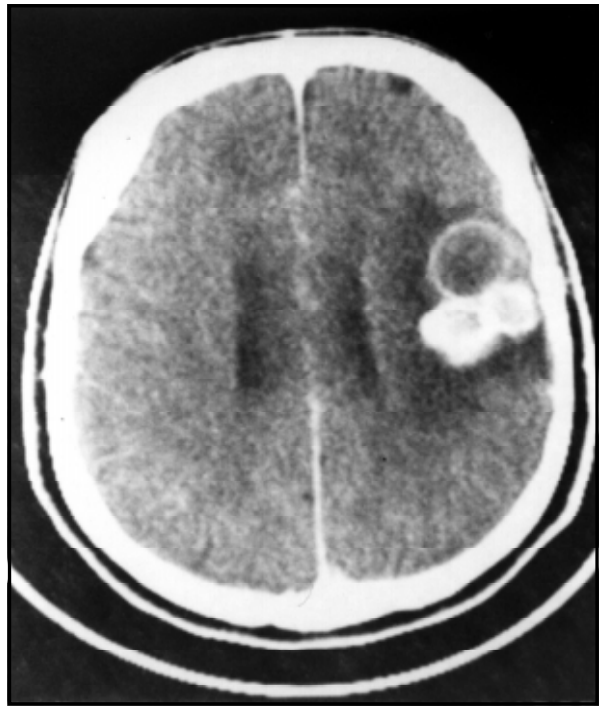

Fig 3. Caso 1. TC de crânio (10/01/88) mostrando regressão parcial e calcificação de algumas das lesões após 2 anos.

após 90 dias, teve alta hospitalar em uso de tuberculostáticos e corticóide. TC de crânio (10/01/86) mostrou desaparecimento das lesões frontais e aumento do tamanho das lesões parietais (15mm) (Fig 2). Após 2 anos, TC de controle (10/01/88) mostrou regressão e calcificação das lesões (Fig 3). O paciente mantém-se assintomático.

Caso 2. Paciente do sexo feminino, 31 anos, manicure, mulata, natural e procedente de Salvador, admitida no Serviço de Emergência do Hospital Espanhol em junho de 1990 com tosse produtiva, perda de peso, astenia e febre baixa há 90 dias. Ao exame físico apresentava-se lúcida, emagrecida, febril, taquipnéica, com estertores crepitantes finos difusos e bilaterais, hepatomegalia e púrpura nos membros superiores. Baciloscopias foram

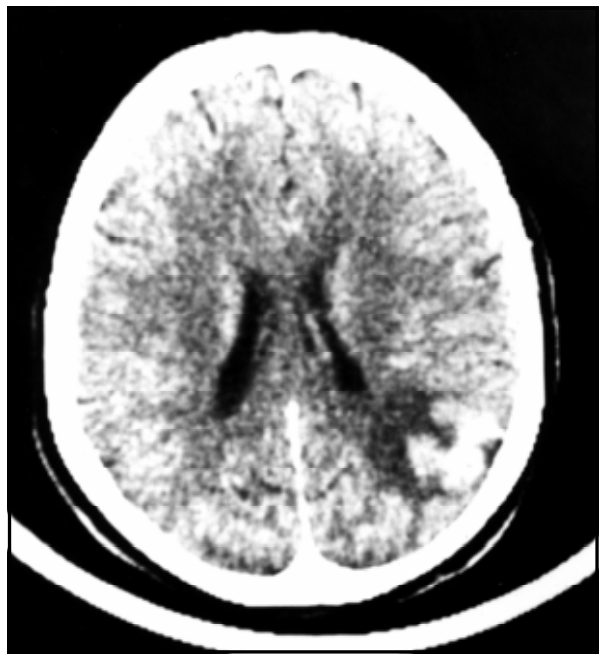

Fig 4. TC de crânio (08/11/90) mostrando presença de lesões nodulares hiperdensas com edema perilesional parieto-occipital no curso do quinto mês de tuberculostáticos.

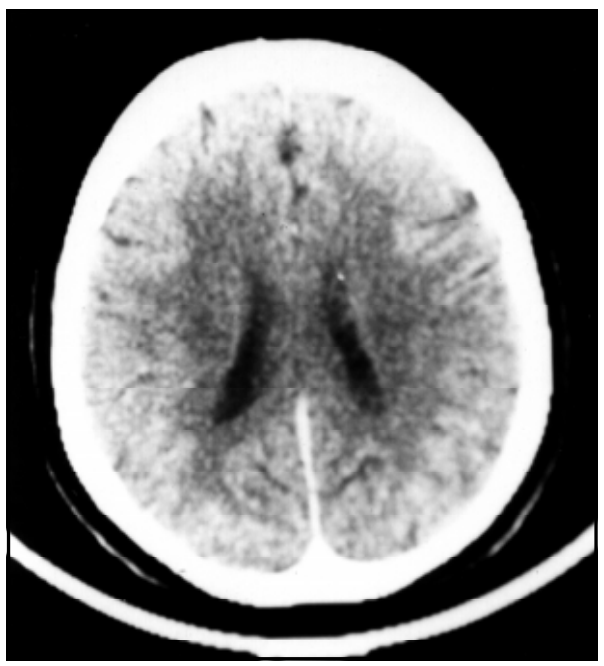

Fig 5. TC de crânio (22/10/91) mostrando desaparecimento das lesões após introdução do corticóide. 


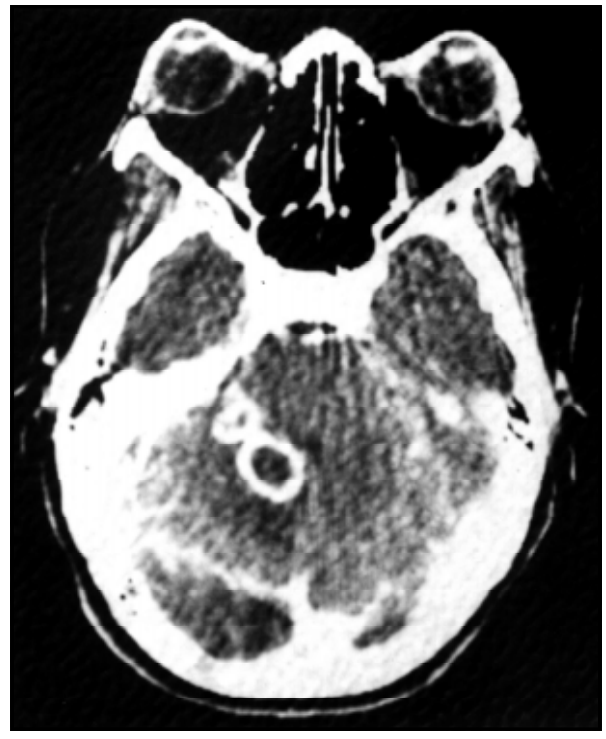

Fig 6. Caso 3. TC de crânio (08/07/91) mostrando 2 lesões areolares no hemisfério cerebral direito, próximo à linha média após 30 dias de tuberculostáticos para tratamento de tuberculose meníngea negativas; o RX de tórax mostrava nodularidade difusa bilateral. Foi iniciado esquema terapêutico com RMP, INH, PZA para tratamento de tuberculose miliar. Com 30 dias apresentou melhora clínica importante. No quinto mês de tratamento ambulatorial apresentou dois episódios de crises convulsivas, sendo realizada TC de crânio (08/11/90) que mostrou lesão de $5 \mathrm{~mm}$ na região occipito-parietal esquerda com importante halo de edema perilesional (Fig 4). Foi acrescentado corticóide à terapêutica, evoluindo com melhora neurológica, e após 30 dias teve alta hospitalar. TCs de crânio posteriores mostraram diminuição da lesão, e 11 meses depois seu completo desaparecimento (Fig 5). Paciente permanece assintomática.

Caso 3. Paciente do sexo feminino, 35 anos, preta, natural e procedente de Salvador, foi internada no Hospital Universitário Prof. Edgar Santos em 03/ 08/91 com queixa de cefaléia e tontura rotatória importante. Ao exame físico apresentava sinais de irritação meníngea e paralisia do VI nervo craniano esquerdo. Realizou estudo do líquor, que mostrou celularidade aumentada com linfocitose importante, glicose baixa e pressão aumentada. A cultura do líquor foi positiva para BK. Foi iniciado tratamento com tuberculostáticos, vindo a apresentar melhora do estado geral, porém mantendo sintomas neurológicos. Foi realizada TC (08/07/91) que mostrou duas lesões areolares de 3 a 5 mm de diâmetro, com halo captante no hemisfério cerebral direito, próximo à linha média (Fig 6). Foi instituída corticoterapia com regressão dos sintomas, tendo alta hospitalar com 20 dias. A paciente não retornou para controle tomográfico.

\section{DISCUSSÃO}

Relatamos três pacientes com acometimento do SNC pela tuberculose durante o uso de tuberculostáticos. Os três pacientes tinham idade superior a 20 anos, apesar de a literatura mostrar que o acometimento do SNC é mais comum em crianças ${ }^{1}$. Dois deles apresentavam tuberculose pulmonar, sendo um miliar, e o outro apresentava comprometimento meníngeo. Os três casos apresentaram melhora do estado geral após introdução da quimioterapia, aparecendo sintomas neurológicos focais entre 60 e 150 dias, quando evidenciadas lesões compatíveis com tuberculomas. O Caso 2 apresentava discreta calcificação do nódulo, e o Caso 3 apresentava anel calcificado. No Caso 1, observou-se aumento da lesão de 10 para $15 \mathrm{~mm}$ quando iniciado o tratamento. Após introdução de corticoterapia houve regressão parcial e calcificação da lesão ${ }^{3}$. No Caso 2,com lesão menor, houve regressão total da alteração à TC, no controle de 11 meses $^{5,6}$.

O acometimento de nervos cranianos foi observado em 15 a $30 \%$ das crianças com tuberculose meníngea ${ }^{7}$. No nosso estudo foi encontrada paralisia do VI nervo craniano em um paciente com tuberculose meníngea e posteriormente ao desenvolvimento de tuberculomas. Neste caso não houve oportunidade de confirmar a regressão anatômica da lesão, apesar da melhora clínica.

Acreditamos que nossos casos coincidem com aqueles da literatura britânica ${ }^{2}$, reforçando em nosso meio indicação do acompanhamento tomográfico em pacientes com ou sem tuberculose pulmonar que apresentem manifestações focais de comprometimento do SNC.

Na tentativa de explicar o fenômeno da expansão paradoxal das lesões cerebrais na vigência do tratamento, similarmente ao que sabidamente ocorre com gânglios linfáticos, sugerimos que esse fenômeno possa se dever à melhora do estado imunológico do paciente com maior hipersensibilidade, o que levaria, além do edema perilesional sugerido por Chambers et al., a entumescimento das 
lesões provocado pela liquefação do respectivo centro² ${ }^{2}$ No entanto, maior número de estudos nessa área é necessário para melhor entendimento desse fenômeno.

\section{REFERÊNCIAS}

1. De Angello M. Intercranial tuberculosis: case report and review of the literature. Neurology 1981,31:1133-1136.

2. Chambers ST, Rudge P. Paradoxal expansion of intracranial tuberculosis: a CT study during chemotherapy. Lancet1994;2:181-184.

3. Tandon NP, Bhargava S. Effect of medical treatment in intracranial tuberculosis: CT studies. Tubercle 1985;66:85-97.

4. Traub M, Kimgrany HPD, Sarah M. Tuberculosis of the central nervous system. Q J Med, 1984;20:81-100.

5. Stead WW, Jordahl W. The clinical spectrum of primary tuberculosis. Ann Intern Med, 1968;68:731-745

6. Humphder JM, O'Mahaay GS. Sympthomatic intracranial tuberculoma. developing during treatment of tuberculosis: a report of 10 patients and review of the litterature. Q J Med, 1987;241:440-460

7. Lincoln EM, Sordillo SVR, Davies PA. Tuberculous meningitis in children: a review of 167 untreated and 74 treated patients with special reference to early diagnosis. J Pediatr 1960;57:807-823 